Fecha de recepción: julio 2021

Fecha de aprobación: agosto 2021

Fecha publicación: septiembre 2021

\section{La Calle, un Territorio con otras prácticas, otros saberes y otros sentidos}

Andrea Forero Hurtado ${ }^{(1)}$ y Andrés

Torres Poveda ${ }^{(2)}$

Resumen: Tras una intervención de la alcaldía de Bogotá en 2016 a una zona del centro de la ciudad, deprimida por fenómenos de narcotráfico, prostitución, miseria e ilegalidad, conocida como El Bronx o La Ele, se desarrollaron acciones para la atención de las personas que ocupaban el sector; algunas centradas en el arte, como la obra de teatro musical "Gloria, un canto a la vida". De allí la investigación "Del territorio de la calle al territorio de la escena teatral”. La riqueza de los relatos sobre las prácticas y los saberes de La Calle como territorio, es decir como espacio de la construcción de sentidos, dieron pie a este artículo. El tema principal en este escrito, antes que el hecho teatral, es el fenómeno de habitabilidad de calle descubierto a través de las narrativas y que suscitaron reflexiones y tímidas propuestas de manejo.

Palabras clave: territorio - calle - habitabilidad de calle - saberes - sentidos - narrativas sistematización.

[Resúmenes en inglés y portugués en las páginas 104-105]

(1) Andrea Forero Hurtado. Comunicadora social-Periodista (Universidad Externado de Colombia). Magister en Comunicación Educación en la Cultura (Uniminuto) y en Comunicación Educativa (Universidad Tecnológica de Pereira) y estudiante del Doctorado en Comunicación (Universidad Nacional de La Plata). Docente e investigadora en la Facultad de Comunicación Social y Periodismo. UNIMINUTO. Coordinadora del GT en Arte y Política CLACSO. Mail: andreadelpforeroh@gmail.com

(2) Andrés Torres Poveda. Antropólogo (Universidad Nacional de Colombia). Magíster en Gerencia Social (Uniminuto). Coordinador en Área de Cultura y Paz. Fundación Arte y Comunicación Vitae y Profesional del Grupo especial PDET-ESAP. Miembro del GT en Arte y Política CLACSO. Mail: atorrespv@gmail.com 


\section{Introducción}

El 28 de mayo de 2016 un grupo de 2.250 policías, 320 soldados, 350 miembros del CTI (Cuerpo Técnico de Investigaciones, un órgano de la Fiscalía General con funciones de investigaciones judiciales), 12 fiscales especializados, 120 funcionarios del Instituto Colombiano de Bienestar Familiar - ICBF, 80 de la Secretaría de Integración Social y 60 funcionarios de la Secretaría de Salud, irrumpieron en una zona del barrio Voto Nacional, ubicado en pleno centro de la ciudad y a pocas cuadras del Palacio Presidencial y la Alcaldía de Bogotá (El Tiempo, 2018). Se trató de una intervención radical encabezada por la misma alcaldía a un sector denominado El "Bronx" o "La Ele", el cual se encontraba saturado de hechos delictivos como el tráfico de estupefacientes, prostitución ilegal, explotación infantil, homicidios, incluso secuestros, y se había constituido en una especie de tierra de nadie, heredera de un proceso similar de intervención y desalojo efectuado casi veinte años atrás en un sector vecino llamado "El Cartucho".

Después de este hecho se sumaron algunas líneas de acción a las ya existentes en cabeza de la Secretaría de Integración Social (SDIS) y el Instituto Distrital para la Protección de la Niñez y la Juventud (IDIPRON) para la atención de personas en situación de habitabilidad de calle destinadas específicamente para las personas que en ese momento ocupaban ese sector, dentro de las cuáles nació el montaje de "Gloria, un canto a la vida", una obra teatral y musical protagonizada por más de ochenta jóvenes, algunos ex habitantes de calle y otros que por diferentes razones estuvieron en riesgo de habitarla ${ }^{1}$.

A partir de esta obra se desarrolló una investigación que se planteó como una sistematización de experiencias del momento particular en que se construye y desarrolla la puesta en escena, dejando de lado los análisis alrededor de la institucionalidad que la promovió, las políticas públicas, las expectativas a futuro, etc., de modo que el momento vital de la investigación es en el cual las y los jóvenes vivieron su propia experiencia individual y colectivamente y para este escrito se tomará el fragmento dedicado a la calle como territorio. Ahora bien, cuando hablamos aquí de sistematización nos referimos a la posibilidad de generar otras maneras de conocimiento apartadas a las instauradas dentro del marco amplio de lo que se denomina genéricamente como Occidente, con lo que cambia el valor político y por ende la posibilidad de la transformación y liberación, lo cual se inscribe metodológicamente como una sistematización de experiencias, siguiendo la definición de Marco Raúl Mejía². Así, la sistematización se presenta como una forma de construir conocimiento a través de la práctica, entendiendo que ésta tiene un valor histórico y que es un proceso complejo en el que participan diversos actores situados en contextos socioeconómicos determinados.

El texto expuesto aquí está basado en la investigación "Del territorio de la calle al territorio de la escena teatral", específicamente en su tercer eje de sistematización "El Contexto", el cual explora las prácticas y los saberes de las y los jóvenes como actores sociales en "la calle", a partir de sus propias narrativas y de las canciones compuestas para la obra teatral. Es necesario aclarar que este escrito se refiere a la vida que llevaban estas personas en la calle y que sirvió como materia prima para la obra de teatro mencionada y que la construcción teatral y las transformaciones que produjo serán insumo para futuros escritos. En esta ocasión, lo interesante es pensar la calle como un territorio que supera la definición del 
lugar físico y se constituye como un espacio de construcción de sentidos, de interacciones simbólicas, de aprendizajes y de relaciones de poder, que suele ser menospreciado o invisibilizado por la sociedad que podríamos denominar hegemónica.

\section{Aproximaciones al concepto de Territorio}

El territorio se puede concebir como un espacio dotado con sentidos, significados y saberes, para una comunidad específica lo cual lo faculta como un medio que contribuye a tejer sociedades donde se logra tener una construcción subjetiva e intercambio de pensamientos y conocimientos entre sus habitantes.

Para que el territorio tenga sentido es indispensable que sea habitado por una comunidad de personas, quienes le dan otra perspectiva a la zona convirtiéndola en una porción terrestre con significado; en palabras de Mario Sosa "Es, sobre todo, un espacio construido socialmente, es decir, histórica, económica, social, cultural y políticamente” (Sosa, 2012, p. 7). Todo lo anterior determina la historia de la comunidad y genera un tejido social donde las personas se reconocen, se ayudan y se fortalecen.

Los actores de la comunidad tienen representaciones sociales dentro de su territorio y dimensiones simbólicas del mismo; por ejemplo, los espacios que los identifican, los que están cargados de temor, las fronteras y límites, o los lugares que les son permitidos o prohibidos, ya sea por entidades gubernamentales o por otros actores dentro de la sociedad. De esta manera, se ve como "el territorio como lugar de encuentro, pero también como lugar de representación, puede ser "vivido", "experimentado", "habitado" de diversas maneras y, por ende, "relatado" de modos diversos" (Algranati, Bruno, \& Iotti, 2012, p. 10). No todas las personas tienen la misma representación de un espacio determinado, puesto que cada una experimentó cosas en diferentes momentos y territorios, esto llevará a cada individuo a hablar de diversas maneras sobre su experiencia en un determinado lugar.

Se empiezan a hacer evidentes las prácticas comunicativas de enunciación, ya que cada sujeto tiene pensamientos y sentimientos únicos, además una memoria compartida generada por la cultura, esto lleva a que cada persona cuente o hable del territorio desde su subjetividad, puesto que cada uno lo entiende desde su geografía, concepciones, percepciones, evaluaciones y expresan su conocimiento con determinadas palabras dadas por una cultura heredada. El territorio crea un vínculo afectivo con cada persona que lo habita, ya sea porque creció allí, porque su familia vivió ahí, porque fue donde estudio, trabajo o formó un hogar. Estas vivencias le producen al individuo un sentido de pertenencia, que no se tiene con ningún otro espacio; estos otros lugares Marc Augé los denomina los no lugares, es decir, espacios que no tienen ningún valor simbólico (Augé, 1992). Los territorios se convierten en parte de la historia de las personas, entonces dejan de ser un terreno común y pasan a tener un valor emocional.

"El territorio, a diferencia del espacio físico, se transforma permanentemente en una serie de significaciones culturales con implicancias históricas y sociales" (Carballeda, 2015, p. 2). Los territorios a través de la historia, por los acontecimientos que ocurrieron dentro de su espacio, se empiezan a transformar y para que las personas no pierdan su cultura 
amoldan lo nuevo a lo que tenían, es decir a pesar de los factores que las puedan afectar ya sea en el aspecto físico, económico o social, lo asimilan y lo apropian, buscando la manera de no perder su esencia.

Cada comunidad tiene representaciones únicas, una cotidianidad naturalizada, formas de organización y tensiones entre ellos o con agentes externos; la rutina se naturaliza y las acciones se vuelven repetitivas. Cada una de estas cosas deriva en que las sociedades conformen su propia cultura, alrededor de una práctica social que se ve reflejada en cada espacio geográfico de diversas maneras; "En este sentido, el territorio como construcción social se explica desde la complejidad del ser humano y esa complejidad es lo que se intenta recuperar" (Sosa, 2012, p. 5). No todas las personas o comunidades tienen las mismas representaciones o formas de vivir, no hablan de la misma manera, tampoco tienen los mismos símbolos o significados, por ello cada territorio debe entenderse desde diferentes puntos de vista y para lograrlo es necesario acercarse a la comunidad e identificar cuáles son sus prácticas culturales.

El territorio es un espacio que permite generar un lugar de encuentro para muchas comunidades, donde se puede apreciar la multiculturalidad, lo cual conlleva a que sea visto

Como una construcción integral, dialéctica, compleja, multidimensional y pluridimensional, desde la vida social y sus múltiples y plurales interrelaciones, procesos y dinámicas, donde lo geográfico y ecológico, lo económico, lo social, lo cultural y lo político fueran entendidos como partes indivisibles y en interacción, lo mismo que sus niveles, ámbitos y escalas (Sosa, 2012, p. 2).

\section{La Calle como Territorio}

Ahora bien, de acuerdo con lo dicho, habitar la ciudad es enfrentarse a un espacio con múltiples significaciones, en el que coexisten diversos grupos sociales que de manera fragmentada constituyen territorialidades heterogéneas en los que los ciudadanos se ubican de acuerdo con sus necesidades. Así, las personas habitantes de la calle se sitúan en un lugar dependiendo de las urgencias del momento en un ejercicio de apropiación del territorio, rompiendo los imaginarios de amenaza y miedo alrededor de la calle y resignificándola como un espacio de protección, especialmente cuando son jóvenes que se sienten rechazados por sus familias o que no recibieron en su casa. Esta situación la ejemplifica la opinión de una de las personas entrevistadas:

Si en tu casa es donde te vulneran, si en tu casa es donde te maltratan, es donde no te quieren es muy fácil que cualquier persona caiga a la calle ¿y en la calle qué vas a encontrar? Pues sí, vas a encontrar frío, hambre, pero va a haber alguien que por algún motivo se va a acercar a ti, y lastimosamente los que están en la calle y sobre todo los que deambulan en la noche que no son, pues entre comillas, las mejores personas, sino que a ellos también les ha tocado empezar a sobrevivir en la calle, pero es el tema de que yo te cuido. Entonces es el tema 
entrar a cualquier persona que te ofrezca ese cuidado, esa protección que en tu casa no te están dando, pues es muy fácil que te quedes ahí y que des la vida por una persona que puede que te esté explotando, que puede que te robe, que puede que acabe con muchas cosas en ti, pero es quien te está cuidando, quien te está protegiendo. Entonces encontrar eso, digamos, es negativo en la calle, también te engancha; estar ahí es encontrar una familia. Creo que nosotros todo el tiempo estamos buscando, una familia y nuestra familia se convierte en nuestros amigos, en los vecinos o el compañero del trabajo pero siempre estamos buscando una red, y si la red yo la encuentro en donde está la droga, está la corrupción, donde está todo lo malo seguramente me voy a quedar allí (Participante 17, comunicación personal, 22 de marzo de 2018).

La misma fuente complementa el panorama mostrándonos cómo la calle tiene más de una cara y constituye un territorio complejo de definir:

(...) sí, la calle también se presta para muchos ambientes sanos, pero es eso, pero es buscar que el ambiente sí sea sano para estar allá. La calle no es un monstruo, ese monstruo lo convertimos nosotros y a quienes nosotros hemos rechazado para que se conviertan en eso, o sea todo juega como una cadena. Entonces la calle también pude ser diversión y muchas veces ellos también encuentran esa diversión y encuentran pues cosas que les gusten allá afuera que muchas veces no son las más sanas, pero es eso, es mostrar que hay una parte divertida de la calle (...) y son chicos que por su condición que por haber sido habitantes de calle, pues ya, ese se vuelve su hogar y ya nadan como pez en el agua y ya saben por dónde meterse y ya saben dónde queda, por donde es, su creatividad también para sobrevivir en el mundo les ha permitido eso, entonces también es un tema, no es que sea buena ni mala sino que todo depende del contexto que uno la quiera ver (Participante 17, comunicación personal, 22 de marzo de 2018).

Definir el territorio implica entonces referirse al lugar de las interacciones sociales, donde hay estructuras de poder, de conflicto y de solidaridad. En este espacio la apropiación está mediada por la permanencia en un sector definido, lo cual va en contravía con una de las características de la población que habita la calle: el tránsito permanente. En efecto, la indagación muestra cómo algunos de los habitantes de calle se mantienen en un mismo espacio y otros son nómadas urbanos que normalmente no crean lazos duraderos con los espacios.

Si tenemos entonces que el territorio resulta de la relación entre el espacio geográfico y los sujetos que pertenecen a una comunidad, se puede afirmar que la calle es el territorio de esta población, pues es allí donde se generan sus interacciones y en donde adquiere los significados, saberes y prácticas para el desarrollo de su cotidianidad. La diferencia de estas personas "marginadas" con el resto de la sociedad, es que el territorio que habitan como su entorno privado es público y al mismo tiempo itinerante, informal y efímero, un espacio que puede llegar a consumir a quienes lo habitan; en la obra de teatro hay consciencia 
de la fuerza de la calle y lo evidencian por medio de la canción "Gorrión” (Construcción Colectiva, 2017).

Un hermano tenía, era un ángel de Dios

pero la vida en el pueblo a él lo cansó

Coro

Voló como un gorrión, la ciudad se lo tragó, perdido lejos de su nido la calle a él lo consumió

perdido lejos de su nido la calle a él lo consumió

Un amor también tenía, a la guerra marchó,

no sé qué será su vida, lo cierto es que dios me lo quitó

espero que la vida regrese lo que quitó

Que la tristeza en el pecho desaparezca con el viento,

Que la tristeza en el pecho desaparezca con el viento

De acuerdo con los relatos de las y los jóvenes que se abordaron, escoger un espacio para permanecer depende de que éste sea seguro, que se pueda trabajar en algo o se puedan satisfacer las necesidades básicas. Estas son las características que hacen que las personas que allí viven consideren que es habitable, a pesar que no cuente con las condiciones mínimas para una vida digna para esta población trashumante y desarraigada. Los sentimientos para estas personas alrededor de la calle son contradictorios, pues por un lado reconocen lo dura que puede llegar a ser y por el otro valoran las posibilidades de vida que les dio, como se refleja en sus propias palabras; "porque la calle cansa por mejor que sea la vida. Por la indiferencia de las personas, por el frío, por el hambre, por muchas cosas" (Participante 14, comunicación personal, 07 de junio de 2017), lo cual contrasta con la frase de otro de los indagados "La calle es el lugar donde han encontrado muchas veces ese refugio que necesitan" (Participante 12, comunicación personal, 07 de junio de 2017).

De hecho, la forma en la que estas personas, entienden la calle es muy diferente a los privilegiados que no viven esa situación, pues mientras para los ciudadanos con casa la calle es un espacio inseguro y amenazante del cual hay que protegerse, para muchos de quienes la habitan es un espacio de libertad, creatividad e incluso de protección. Esto se evidencia en la letra de la canción "Salsa a la calle", que fue cantada por los artistas de la calle en "Gloria".

\section{Coro}

La calle me gusta, la calle es así,

En la calle soy libre ¡Ay! Que calle feliz (bis)

En la calle soy libre esa es mi felicidad,

No necesito un rifle, quiero una oportunidad

Me gusta la calle, que baile mi gente

Que baile en la calle hasta el presidente

Comprender el concepto de "la calle" como territorio implica identificar las prácticas y saberes de aquellos que la habitan, en este caso, las historias de vida de las y los jóvenes participantes de esta experiencia se caracterizan por las carencias físicas y emocionales 
así como por la resistencia a las figuras de autoridad. Estas son las razones recurrentes del que la mayoría de ellos permanecieran en la calle, si bien en algunos casos lo hicieron por vivir una especie de aventura, ya sea solos o con amigos. En algunos casos este fue el modus vivendi que heredaron y en otros pocos una forma de salvarse, como lo expresó un entrevistado: "Mi mamá me dio la plata para que me fuera, a mí ya me andaban buscando los policías y la gente que yo también le debía plata, tocaba irse de ahí” (Participante 04, comunicación personal, 03 de diciembre de 2016).

\section{La vida en "la calle"}

En cuanto a su procedencia, la mayoría de las personas integrantes de este grupo de jóvenes, no nacieron ni crecieron en la calle, sino que vienen de familias de escasos recursos o con condiciones de disfuncionalidad que, voluntariamente o no, incidieron en la decisión de irse a recorrer otros espacios. De hecho las problemáticas familiares suelen ser el centro de las historias de vida de los actores sociales y comúnmente son causa y consecuencia de que se piense en la calle como una opción y se convierta en un lugar de construcción/ producción de conocimientos desde la vida cotidiana.

En cuanto a la vida en la calle, los relatos explican que el sitio en el que vivían estaba dispuesto como un espacio personal y privado al que no se deja entrar a cualquiera; solía ser un cambuche que, aunque se percibe como un terreno de independencia, distanciado de otros, se articula dentro de un sentido de comunidad y de códigos de comunicación definidos con otras personas que cuentan con condiciones similares de vida. Sin embargo, en esta "nueva construcción de comunidad", se retoman aspectos de aquella otra sociedad, la tradicional, y se resignifican acorde con la nueva situación de vida. Las personas entrevistadas comentaron que no era fácil compartir el espacio de dormir, pero que una vez aceptaban al otro, se creaba una relación de solidaridad, especialmante con los nuevos en la calle; en consecuencia son recurrentes testimonios como el siguiente en el que además se comparten adicciones:

Yo dormía en la calle, entonces ponle cuidado, que yo dormía en los parques abandonados, ehh... de esos que son de madera y que tienen un tobogán. Pues un día eran como las tres de la mañana llegó un chino y llego con todas las cosas y me dijo que lo habían sacado de la casa, yo ni conocía al chico y el chino a lo que me vio me dijo "déjeme dormir ahí", yo le dije que pero “¿cómo lo voy a dejar dormir si solo cabe uno socio?” y entonces ponle cuidado que él tenía la de -muchas-cosas, tenía hasta una mata, entonces se quedó ahí afuera con las cosas y a mí me dio visaje. “¿Usted echa Gale? ”” y el chino me dijo que sí y comenzamos a galearnos. Me dijo que tenía la de -muchos- problemas, entonces le dije que si se iba a chirriar o qué; el chino me dijo que no (Participante 09, comunicación personal, 06 de diciembre de 2016). 
En cuanto a las necesidades básicas, según los relatos, éstas se proveían a través de actividades como el reciclaje, el cuidado de carros, limpieza de vidrios, la mendicidad, el cosquilleo y en el caso de algunas jovencitas, la prostitución, oficios aprendidos por medio del ejemplo al ser integrados a grupos y cadenas de economía informal legal e ilegal. Hay que resaltar que a pesar que las y los jóvenes asuman eventualmente nuevos roles sociales apartados de los desempeñados en "la calle", siguen siendo estigmatizados e invisibilizados desde la que, desde una perspectiva cultural hegemónica, es asumida como la sociedad normal.

Por lo contrario, en el momento de ingresar al contexto de ese nuevo entorno de la calle fueron y son reconocidos, al tiempo que reconocen a los otros con quienes comparten la vida, incluso despiertan un sentido de pertenencia. Comprender su contexto nos permite descubrir "otra sociedad o comunidad", formada por habitantes de calle, jíbaros, prostitutas, proxenetas, vecinos o comerciantes de las zonas, etc., en la cual cada quien construye relaciones sociales basadas en la cooperación, en la protección o algunas veces en la explotación.

Muchos de los indagados mencionaron que el principal detonante del desarrollo de su vida en la calle era el consumo de sustancias psicoactivas (SPA) tales como marihuana, bazuco y pegante entre otros, a los que llegaron después del alcohol y el cigarrillo como una forma de evasión de los problemas familiares anteriormente mencionados, generando una adicción difícil de manejar. Esto, según sus relatos, los llevó a hacer cosas que antes no hubieran hecho, "pues porque usted le dan esas ansias de fumar y le da como una cosa, de un 'uyy', eso me sirve pa la fuma, voy y me lo robo, me lo guardo y me lo robé y ya, tengo pa cuantas bichas ${ }^{4}$, un bareto, cigarrillos, una pipa" (Participante 10, comunicación personal, 15 de junio de 2017). Otro de los actores nos contó,

Eso es una mierda créalas, eso el que es drogadicto, paila ${ }^{5}$, eso vive mucho menos, paila, lo tratan a uno mal... Eso uno hace muchas cosas por una bolsa de bazuco... hasta roba, hasta dice mentiras, hasta le quita a los seres queridos y lo peor es que uno pierde todo, uno pierde la familia, pierde los amigos, pierde las oportunidades (Participante 07, comunicación personal, 06 de diciembre de 2016).

Según sus testimonios, la apatía y la agresión se convirtieron en sentimientos constantes en sus historias de vida. Estas aflicciones los empujaron a la calle en donde conocieron amistades que les enseñaron a sobrevivir en ella, transmitiéndoles los conocimientos necesarios en ese tránsito del "sufrimiento" de la casa a la "tranquilidad" de la calle. Por supuesto en este trasegar abandonaron el colegio y muchos entraron en las cadenas de la delincuencia y la prostitución y subsecuentemente llegaron los embarazos, los abortos, las enfermedades sexuales, los centros de rehabilitación, las venganzas, etc. Al poco tiempo fueron personas diferentes a las que eran, cambió su personalidad y su pensamiento, mostrando dificultad para interactuar con otros y para desarrollar las actividades de la vida diaria y se insertaron en un ciclo que les generó cada vez mayores exclusiones. Bajo esta perspectiva, las y los jóvenes que habitan la calle son vulnerables, están expuestos a las dinámicas de la delincuencia y el abuso, son proclives a la conformación de pandillas 
buscando pertenencia y protección ante su baja estima, autoconfianza y el descuido por parte de la sociedad.

Ante esto, un elemento importante a resaltar es que a pesar que habitar la calle pareciera ser sinónimo de soledad, allí se crea este sentido de comunidad dado a partir de la creación de dispositivos que permiten delimitar esos espacios comunitarios. Los elementos que los constituyen cuentan con significados que se enuncian y se narran entre ellos mismos, la fogata, la basura, etc.; así pues, quien vive en la calle cuenta con unas competencias comunicativas que nos son ajenas a aquellos que no pertenecemos a ese entorno, crean códigos que demarcan incluso las llamadas fronteras invisibles, es decir, restringen el paso a quienes no son bienvenidos. Así, vemos cómo estos marginados cuentan con un particular sentido de identidad, reconociéndose como habitantes de calle.

En cuanto a este aspecto, hay que mencionar que algunas personas entrevistadas contaron cómo en la calle las mascotas muchas veces constituyen "la familia". Desde su condición de excluidos, ni el animal callejero, ni el habitante de calle existen para el sistema y cuando se nombran, tanto los unos como los otros, se referencian como "problemas" y en términos de cifras relacionadas con la necesidad de mitigación o la disminución. Al ser indagados por sus familias varias de las personas entrevistadas manifestaron no tener o no saber nada de ellas, algunos en actitud de resentimiento y otros de apatía por el tema. Este desinterés se manifiesta de manera especial con las y los jóvenes rechazados de sus núcleos familiares por sus condiciones especiales, como por ejemplo, el de ser transgénero, característica que en la calle no es motivo de discriminación, por lo menos en algunos círculos, a diferencia de otros contextos como el del barrio o la familia.

Es interesante observar que para los actores de esta investigación la autoridad representa represión y no creen en lo que consideran la Política, el Estado, la alcaldía. No obstante, parecen sentir afecto sincero y agradecimiento por los funcionarios distritales que los abordan constantemente y que llegan con campañas de salud, higiene, protección, etc., como si se tratara de favores personales más que misiones institucionales. De hecho, en algunas ocasiones esas relaciones traspasan lo profesional y se constituyen en amistad; en palabras de uno de los actores sociales:

Mi mensaje sería dejarnos educar por estos excelentes educadores que en realidad después que pasa el tiempo ya tu no los ves como educadores sino como el compadre, el pana, el socio, hasta el papá y pues la verdad pues dejarnos educar ya que ellos alcanzaron a conocer a ese padre lindo, Javier de Nicoló, que les enseñó muchas cosas y la verdad así mismo ellos nos han dado para salir con nuevos proyectos hacia la sociedad, y pues lo que dijo el profesor, aunque ha cambiado mucho se trata de recuperar; es muy difícil, pero pues se trata de recuperar. Entonces para mí, valoro mucho a esos profesores porque ellos alcanzaron a recibir ese amor del padre Javier de Nicoló y sé que en ellos también está ese padre que esta descansado (Participante 23, comunicación personal, 14 de marzo de 2018). 
Por otra parte, la evidencia de la mala imagen de la autoridad se hace patente en una de las canciones de la obra, que se llama "Todos corran" (Espitia, 2017), inspirada en el desalojo de la calle del Bronx:

Todos, todos corran no se dejen atrapar, que la tomba viene no se dejen agarrar, Pillaron tres caletas, esta cosa está sería, el que vendía vareta se quedó en la miseria.

Esto es un infierno nos echaron de este valle, que ahora todos recen nos mandaron pa otra calle.

Nos sacaron del Palacio ahora todo el mundo grita fuerte $\mathrm{y}$ ahora quien sabe donde podemos estar.

Todos todos corran no se dejen agarrar, todos todos corran no se dejen atrapar, todos todos corran no se dejen agarrar, que todos aquí vamos a volar.

\section{El valor del Relato}

En cuanto a su forma de comunicar, se puede decir que estas personas valoran de manera especial la oralidad, desde la palabra narran sus historias de vida, de una manera a la vez irónica y sentida, aunque el pasado les adeuda, también es él quien les permite vivir por medio de sus narraciones. De no ser por la palabra hablada muchos de ellos no hubieran sobrevivido a la calle, pues fue gracias a las historias de sus parceros o vecinos de cambuche que aprendieron mucho de lo que saben, en especial el argot de la calle.

Son constantes los relatos de cómo las y los jóvenes manifiestan que se sentían relegados, abandonados, excluidos, en la marginalidad e inferioridad y aún hoy, después de estar fuera de la calle, es evidente que conocen poco acerca de hechos históricos y no les importa lo que sucede en la sociedad, el pasado o el futuro; solo cuentan con las historias que van construyendo en el día a día. La forma en la que se enuncian denota que son conscientes de la exclusión a la que están expuestos, como se revela en otra de las canciones de la obra: "Un amor que se rompió" (Creación Colectiva, 2017):

Este niño medio aindiado

No nos sirve para nada,

Como nada ha estudiado

Lo sacamos a patadas

Este anciano que tenemos

A una muleta se aferra,

Una pierna le debemos,

La ha perdido en la guerra

Coro 
El borracho allí quedo

No se ha recuperado

De un amor que se rompió (Bis)

Una casa yo quería

un poquito de calor

pero un día me fui a la calle

y la calle me atrapo

y de buenas a primeras

me tratan de delincuente

porque vivo yo mi vida

y me comporto diferente

Están acostumbrados a la estigmatización y al prejuicio, lo sienten constantemente y como mecanismo de defensa algunas veces asumen actitudes agresivas y hasta atemorizantes; saben que se les culpa de muchas de las cosas que suceden en la ciudad y son señalados, pero manifiestan que esto no les importa, al punto de que a veces se autoexcluyen. Sin embargo, al hablar de ello se percibe el resentimiento y la necesidad que tienen de incluirse e incluso de ser admirados y, de hecho a algunos la participación en la obra teatral les hace sentirse mejor en ese sentido, como se refleja en el testimonio de una de las personas más activas en el montaje:

Digamos, era una persona jodida en Bogotá, pero de los malos malos, y yo decidí entregar las armas y decir no más, de entregar todo esto, y las personas que me conocen a mi anteriormente me ven ahora y me dicen "marica, yo no puedo creer que sea usted"; yo les digo, la calle te deja a ti una cicatriz. Yo decidí tatuarme el cuerpo para taparme las cicatrices, más de uno me dice "ay que el gomelo, que el ñero"; no, esto para mí es un significado especial, es tapar todo lo malo que me hizo la calle y dar un significado más a la vida y hoy en día le estoy devolviendo el favor por medio de esta obra de teatro (Participante 25, comunicación personal, 14 de marzo de 2018).

\section{La sociedad frente a la habitabilidad de calle}

En cuanto al manejo del fenómeno de la habitabilidad de calle, las políticas que se llevan a cabo tradicionalmente han priorizado perspectivas como la sociológica o la sicológica de acuerdo con la causalidad que se establezca, ya sea de un lado la pobreza, la marginalidad y la falta de oportunidades, o por otro la disfuncionalidad familiar y el maltrato, basándose con frecuencia dichas políticas en el asistencialismo, la religión o la Educación Popular, pero de una u otra manera el albergue es el centro donde se lleva la atención y el objetivo es una pretendida resocialización en el marco de un retorno desde una situación anormal hacia el estado asumido como normal, en términos culturalmente hegemónicos. 
Ahora bien, el problema social que conlleva el fenómeno de habitabilidad de calle se ha abordado desde el postulado que existe una manera correcta de ser sociedad, una sociedad conveniente, normal, que corresponde a una cultura igualmente normal, y con esa base se intenta encontrar la forma de devolver, de arreglar o enderezar la vida de esas personas que se han conformado en comunidades en torno del vivir en la calle. De ahí el término Resocialización o Reintegración.

Sin embargo, la pretensión de una sociedad normal de ésta índole relacionada con la habitabilidad de calle desconoce la complejidad de la situación y la realidad multicultural y profundamente diversa tanto de las sociedades como de los intereses, deseos, historias, de las personas que las conforman y las crean. Por tal razón las propuestas de manejo deberían responder a dicha complejidad; la calle puede pensarse también como espacio de educación y encuentro, considerando competencias que allí se desarrollan y los lazos de cooperación, confianza y solidaridad que se generan. En ese territorio agreste, duro, en el que se viven situaciones peligrosas e injustas, en donde convivir con la ilegalidad no es extraño y en el que se llega al límite en muchas maneras, también se originan saberes propios que tienen que ver con el liderazgo, la comunicación, el argot, la creatividad, la diversidad, las perspectivas múltiples, la justicia, el manejo de las relaciones y los acuerdos, entre otros, de tal forma que resulta recomendable contemplar la posibilidad de ampliar las perspectivas de análisis del tema y de su manejo, de manera que se consideren aspectos como la jerarquía, las construcciones sociales diversas, el multiculturalismo dentro del espacio urbano, etc.

Washington Uranga define la Cultura como un modo de organizar el movimiento permanente de la vida cotidiana, del ser y actuar de todos los días, sencillo, rutinario pero, por ello mismo, configurador de conductas, de modos de ser y actuar. La cultura puede entenderse así como un principio organizador de la experiencia, mediante el cual los sujetos ordenan y estructuran sus acciones a partir del lugar que ocupan en las redes sociales (Uranga, 2007). En consonancia con esta definición consideramos la cultura como una matriz de sentidos previamente definidos dentro de la cual las acciones cotidianas de las personas se codifican y se realiza el intercambio de mensajes que no solo dan significado a la existencia social, sino que a su vez modifican diacrónicamente la matriz.

Así de acuerdo con lo observado, vivir en la calle para muchas de las personas indagadas significa resistirse a los imperativos de la sociedad; el trabajo, el tiempo, la familia, la propiedad y hasta el descanso toman nuevos sentidos. Esto es lo que permite decir que crean "cultura propia" que estas y estos jóvenes no quieren perder, aun siendo conscientes de los sufrimientos y vejámenes que deben soportar.

Tenemos entonces que el análisis de esta experiencia abre la posibilidad de explorar alternativas de acción sobre el problema de la habitabilidad de calle y la marginalidad desde el desplazamiento del énfasis en los parámetros de normalidad y de intervención social, hacia el reconocimiento, no solo de sus implicaciones como problema social, sino de sus características intrínsecas y las potencialidades que guarda, a saber, códigos y formas de comunicación alternativas, conceptos vívidos y fuertes de solidaridad e identidad, de talentos por explotar, y en muchos casos incluso de ansias por ayudar y replicar sus aprendizajes. 


\section{A modo de conclusión}

En este trabajo se pretendió pues, más que comprender el fenómeno de habitabilidad de calle, promover la reflexión sobre la posibilidad de un enfoque ampliado hacia la perspectiva antropológica, complementario a los enfoques sociológicos y sicológicos con que preponderantemente se ha abordado este problema, de modo que a los aspectos económicos, familiares, de crianza, entre otros, se sumen factores como la jerarquía, el simbolismo, las dinámicas de grupo, métodos alternativos de enseñanza y aprendizaje, nuevas formas de participación social y, especialmente, la comunicación.

Desde ese enfoque, una propuesta para el manejo de este fenómeno de habitabilidad de calle, más allá de pensar en "rehabilitar-domesticar" o "hacer olvidar lo aprendido", podría reconocer esos saberes y constituir espacios en el que las personas recuerden lo aprendido y esa "cultura propia" sea la materia prima para la creación de nuevos saberes. Esto constituiría un reto para la comunicación, la educación y la cultura, que tendrían que ser pensadas de manera alternativa, que responda a las necesidades específicas de esta población. De ahí que hacer arte con las personas que habitan la calle, como el caso de "Gloria", se puede ver como una aproximación a este tipo de intervención que sin pretender transformar a esta persona en otra, aprovecha sus saberes desde otros lenguajes como el artístico y propicia la transformación propia.

\section{Notas}

1. "Gloria" será el nombre con el que se hará referencia a la obra teatral de la experiencia relatada en este texto.

2. La sistematización es una forma de investigación que intenta romper esas dicotomías sobra las cuales se ha construido el conocimiento en Occidente: Naturaleza-Cultura, público/Privado, Razón/Emoción, Conocimiento científico/Saber popular, Conocimiento natural/Conocimiento Social, Conocimiento experto/Conocimiento lego, Trabajo manual/Trabajo intelectual y el pilar sobre el que se construye este proyecto, la separación sujeto/Objeto; al pensar estos aspectos como separados, jerarquizados e irreconciliables en donde la producción de conocimiento científico le corresponden a unos individuos denominados investigadores por la posesión de un método y una legitimidad institucional. En cambio, otro saber sería precisamente el que no cumple con sus características de rigor y veracidad (Mejía, 2012, p. 16).

3. En la jerga de la calle al pegamento sintético que se usa para inhalar y que produce efectos psicoactivos se le dice "gale".

4. En la jerga de la calle a un pequeño paquete de drogas psicoactivas se le dice "bicha".

5. En la jerga popular cuando algo o alguien está en malas condiciones, se dice que está "paila". 


\title{
Bibliografía
}

Algranati, S.; Bruno, D. \& Iotti, A. (2012). Mapear actores, relaciones y territorios. Una herramienta para el análisis del escenario social. La Plata: Facultad de Periodismo y Comunicación Social UNLP.

Augé, M. (1992). Los “No lugares". Espacios del anonimato. Barcelona: Gedisa.

Carballeda, A. (2015). El territorio como relato. Una aproximación conceptual. Margen revista de Trabajo Social y Ciencias Sociales, número 76 - marzo 2015 https://www.margen. org/suscri/margen76/carballeda76.pdf

Construcción Colectiva (2017). Gorrión [Grabado por K. Montenegro]. Bogotá.

Correa, C. (2017). Salsa a la calle [Grabado por C. Correa, \& A. Tapias ]. Bogotá.

Creación Colectiva (2017). Un amor que se rompió [Grabado por A. Tapias]. Bogotá.

El Tiempo. (31 de Mayo de 2018). Alcaldía encontró secuestrados y esclavas sexuales en El Bronx. http://www.eltiempo.com/archivo/documento/CMS-16605518

Espitia, D. A. (2017). Todos corran [Grabado por D. Espitia, \& A. Tapias ]. Bogotá.

Mejía, M. (2012). Sistematización. Una forma de investigar las prácticas y de producción de saberes y conocimientos. Vicemeinisterio de educación alternativa y especial Estado plurinacional de Bolivia.

Muñoz, L. (Mayo de 2017). 80 hijos del Bronx montaron una obra para recordar el fin de una olla. https://www.shock.co/cultura-pop/80-hijos-del-bronx-montaron-una-obrapara-recordar-el-fin-de-una-olla.

Sosa, M. (2012). ¿Cómo entender el territorio? Cara Parens.

Uranga, W. (2007). Mirar desde la comunicación. Una manera de observar las prácticas sociales. Facultad de Periodismo y Comunicación Social-UNLP.

\begin{abstract}
After an intervention by the Bogotá mayor's office in 2016 to an area of the city center, depressed by phenomena of drug trafficking, prostitution, misery and illegality, known as The Bronx or La Ele, actions were developed to care for the people who occupied the sector; some focused on art, such as the musical "Gloria, a song to life". Hence the investigation "From the territory of the street to the territory of the theater scene". The richness of the stories about the practices and knowledge of the street as a territory, that is, as a space for the construction of meanings, gave rise to this article. The main theme in this writing, before the theatrical event, is the phenomenon of street livability discovered through narratives and which prompted reflections and timid management proposals.
\end{abstract}

Keywords: territory - street - street habitability - knowledge - meanings - narratives - systematization.

Resumo: Após uma intervenção da prefeitura de Bogotá em 2016 em uma área do centro da cidade, deprimida por fenômenos de narcotráfico, prostituição, miséria e ilegalidade, conhecido como Bronx ou La Ele, foram desenvolvidas ações de atendimento às pessoas 
que ocupou o setor; alguns voltados para a arte, como o musical "Gloria, uma canção para a vida". Daí a investigação "Do território da rua ao território da cena teatral”. A riqueza das histórias sobre as práticas e saberes da rua como território, ou seja, como espaço de construção de significados, deu origem a este artigo. O tema central desta escrita, antes do evento teatral, é o fenômeno da habitabilidade da rua descoberto através das narrativas e que provocou reflexões e propostas tímidas de gestão.

Palavras chaves: território - rua - habitabilidade nas ruas - conhecimentos - significados - narrativas - sistematização.

[Las traducciones de los abstracts fueron supervisadas por el autor de cada artículo] 